\title{
Synthesis, HPLC Enantioresolution, and X-ray Analysis of a New Series of C5-methyl Pyridazines as N-Formyl Peptide Receptor (FPR) Agonists
}

\author{
AGOSTINO CILIBRIZZI, ${ }^{1}$ LETIZIA CROCETTI, ${ }^{1}$ MARIA PAOLA GIOVANNONII, ${ }^{1 *}$ ALESSIA GRAZIANO, ${ }^{1}$ CLAUDIA VERGELLI, \\ GIANLUCA BARTOLUCCI, ${ }^{1}$ GIACOMO SOLDANI, ${ }^{1}$ MARK T. QUINN, ${ }^{2}$ IGOR A. SCHEPETKIN,${ }^{2}$ AND CRISTINA FAGGI ${ }^{3}$ \\ ${ }^{1}$ Università degli Studi di Firenze, Dipartimento di Scienze Farmaceutiche, Via Ugo Schiff 6, Sesto Fiorentino, 50019 Firenze, Italy \\ ${ }^{2}$ Montana State University, Department of Immunology and Infectious Diseases, Bozeman, MT 59717 USA \\ ${ }^{3}$ Università degli Studi di Firenze, Dipartimento di Chimica, Via della Lastruccia 13, Sesto Fiorentino, 50019 Firenze, Italy
}

\begin{abstract}
The synthesis of three racemates and the corresponding non-chiral analogues of a C5-methyl pyridazine series is described here, as well as the isolation of pure enantiomers and their absolute configuration assignment. In order to obtain optically active compounds, direct chromatographic methods of separation by HPLC-UV were investigated using four chiral stationary phases (CSPs: Lux Amylose-2, Lux Cellulose-1, Lux Cellulose-2 and Lux Cellulose-3). The best resolution was achieved using amylose tris(5-chloro-2-methylphenylcarbamate) (Lux Amylose-2), and single enantiomers were isolated on a semipreparative scale with high enantiomeric excess, suitable for biological assays. The absolute configuration of optically active compounds was unequivocally established by X-ray crystallographic analysis and comparative chiral HPLCUV profile. All compounds of the series were tested for formyl peptide receptor (FPR) agonist activity, and four were found to be active, with $\mathrm{EC}_{50}$ values in the micromolar range. Chirality 25:400-408, 2013. () 2013 Wiley Periodicals, Inc.
\end{abstract}

KEY WORDS: liquid chromatography; chiral stationary phase; Lux Cellulose; Lux Amylose; absolute configuration; pyridazinones; FPRs; inflammation

\section{INTRODUCTION}

Inflammation is the first response of the immune system to infection, irritation, and trauma, and it plays a central role in the pathogenesis of many human diseases, such as asthma, rheumatoid arthritis, multiple sclerosis, cancer, and Alzheimer's disease. ${ }^{1}$ The inflammatory response requires tight regulation and control to avoid overshooting, prolongation, and self-induced damage. ${ }^{2}$ Considering that most currently used anti-inflammatory drugs have important limitations, ${ }^{3}$ the extensive search to find immunomodulatory agents that regulate innate immune responses represents a promising strategy for treating inflammation and infectious diseases.

The development of bioactive molecules that selectively stimulate the immune system represents an important challenge in medicinal chemistry. ${ }^{4}$ To this end, the modulation of formyl peptide receptors (FPRs), mediators of key events in the regulation of endogenous inflammation and resolution of inflammation, represents a distinctive approach to control inflammatory events. ${ }^{5}$ FPRs belong to the seven transmembrane domain G protein-coupled receptor (GPCR) family, and three subtypes have been identified in humans (FPR1, FPR2/ALX, FPR3) which exhibit a high level of amino acidic homology. ${ }^{6}$ Being expressed in the majority of white blood cells, FPRs interact with a wide range of structurally different pro- and anti-inflammatory ligands associated with different diseases.

Previously, we identified a large number of potent pyridazinebased FPR1/FPR2 agonists. ${ }^{7}$ Starting from the literature evidence that chiral C4- or C5-substituted pyridazines are generally of particular interest to search for biological activity, ${ }^{8-10}$ as well as reports that stereoselective interaction may enhance the binding with FPRs, ${ }^{11-14}$ we report herein the synthesis of a (c) 2013 Wiley Periodicals, Inc. new series of chiral C5-methyl pyridazine derivatives (compounds ( \pm )-4a-c, Fig. 1) and the corresponding aromatic non-chiral analogues (8a-c) in order to further investigate the role of stereochemistry in their pharmacological activity. Due to the hypothetical stereoselectivity of the drug-receptor interaction, we developed a chiral stationary phases (CSPs)/ eluent system suitable for a semi-preparative scale and performed chiral HPLC-UV separation of optically active enantiomers from each racemates $( \pm)$-4a-c. Analytical methods on four different CSPs (Lux Amylose-2, Lux Cellulose-1, Lux Cellulose-2, and Lux Cellulose-3) were initially investigated to determine the best resolution conditions, and changes in mobile-phase composition were studied to determine the effect of solvents on the column selectivity. On the basis of the preliminary chromatographic results, the resolution of the racemates was subseqently transferred in the semi-preparative system. The absolute configuration of collected optically active compounds was univocally established by X-ray crystallographic analysis of one of the enantiomers in each pair and by comparative elution order during chiral HPLC-UV analysis. Finally, we investigated the pharmacological properties of all racemates, enantiomers and non-chiral anologues as FPRs agonists using in vitro functional assays.

\footnotetext{
Contract grant sponsor: National Institutes of Health; Contract grant number: GM103500.

${ }^{*}$ Correspondence to: Maria Paola Giovannoni, Dipartimento di Scienze Farmaceutiche, Via Ugo Schiff 6, Sesto Fiorentino 50019 Firenze. E-mail mariapaola.giovannoni@unifi.it

Received for publication 23 July 2012; Accepted 11 January 2013

DOI: $10.1002 /$ chir.22162

Published online 6 June 2013 in Wiley Online Library

(wileyonlinelibrary.com).
} 


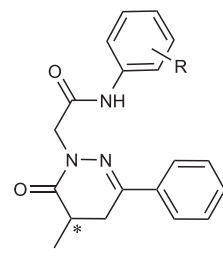

$( \pm)-4 a-c$

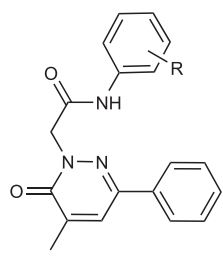

$8 a-c$
$\mathrm{R}=4-\mathrm{Br}, 4-\mathrm{F}$

3,4-methylenedioxy
Fig. 1. Structures of chiral FPR ligands designed.

\section{MATERIAL AND METHODS}

Reagents, starting materials, and HPLC-grade solvents were obtained from commercial sources and used without further purification. Extracts were dried over $\mathrm{Na}_{2} \mathrm{SO}_{4}$, and the solvents were removed under reduced pressure. All reactions were monitored by thin layer chromatography (TLC) using commercial plates precoated with Merck silica gel 60 F-254. Visualization was performed by UV fluorescence $\left(\lambda_{\max }=254 \mathrm{~nm}\right)$ or by staining with iodine or potassium permanganate. Chromatographic separations were performed on a silica gel column by gravity chromatography (Kieselgel 40, 0.063-0.200 mm; Merck) or flash chromatography (Kieselgel 40, 0.040-0.063 mm; Merck). Yields refer to chromatographically and spectroscopically pure compounds, unless otherwise stated. Compounds were named following IUPAC rules, as applied by Beilstein-Institut AutoNom 2000 (4.01.305) or CA Index Name. The identity and purity of intermediates and final compounds was ascertained through $\mathrm{nmR}$, TLC, and analytical HPLC-UV. All melting points were determined on a microscope hot stage Büchi apparatus and are uncorrected. ${ }^{1} \mathrm{H} \mathrm{nmR}$ spectra were recorded with Avance 400 instruments (Bruker Biospin Version 002 with SGU). Chemical shifts $(\delta)$ are reported in ppm to the nearest $0.01 \mathrm{ppm}$, using the solvent as an internal standard. Coupling constants (Jvalues) are given in $\mathrm{Hz}$ and were calculated using 'TopSpin 1.3' software rounded to the nearest $0.1 \mathrm{~Hz}$. Mass spectra $(\mathrm{m} / \mathrm{z})$ were recorded on a ESI-MS triple quadrupole (Varian $1200 \mathrm{~L})$ system, in positive ion mode, by infusing a $10 \mathrm{mg} / \mathrm{L}$ solution of each analyte dissolved in a mixture of $\mathrm{mQ} \mathrm{H}_{2} \mathrm{O}$ :acetonitrile $1: 1 \mathrm{v} / \mathrm{v}$. Microanalyses were performed with a Perkin-Elmer 260 elemental analyzer for $\mathrm{C}, \mathrm{H}, \mathrm{N}$, and the results were within $\pm 0.4 \%$ of the theoretical values, unless otherwise stated.

Analytical HPLC-UV was performed on an Agilent 1200 Series with an autosampler, column oven, and diode array detector (DAD) using chiral Lux Amylose-2, Lux Cellulose-1, Lux Cellulose-2, and Lux Cellulose-3 (50 mm $\times 4.6 \mathrm{~mm}$ I.D., $3 \mu \mathrm{m}$ particle size; Phenomenex, Bologna, Italy) columns. For analytical enantioseparations, the sample solutions were prepared by diluting stock solutions of each racemate at a concentration of $0.1 \mathrm{mg} / \mathrm{mL}$ in the same mixture of solvents used as mobile phase. The injection volume was $10 \mu \mathrm{L}$, the flow rate was $1.0 \mathrm{~mL} / \mathrm{min}$, the temperature of column was $40^{\circ} \mathrm{C}$, and the detector wavelength was fixed at $250 \mathrm{~nm}$. The signal was acquired and processed by Chemstation revision B.03.03-SR2 software. HPLC-grade solvents were supplied by SigmaAldrich (Milan, Italy). The mobile phases tested were mixtures of acetonitrile (MeCN) or $n$-hexane ( $n$-Hex), both with isopropanol (IPA) as polar modifier. The values of retention time $\left(t_{\mathrm{R}}\right)$ are given in minutes.

Semi-preparative HPLC-UV enantioseparations were performed using a Lux Amylose-2 $(250 \mathrm{~mm} \times 4.6 \mathrm{~mm}$ I.D., $5 \mu \mathrm{m}$ particle size; Phenomenex, Bologna, Italy) column. The HPLC apparatus consisted of a Perkin-Elmer (Norwalk, CT, USA) series 200 with a quaternary pump, autosampler, column oven, UV-VIS detector, and Biologic BioFrac fraction collector (from Bio-Rad, Milan, Italy). The column temperature was $40^{\circ} \mathrm{C}$, and the UV detector wavelength was fixed at $250 \mathrm{~nm}$. The signal was acquired and processed by Totalchrom 6.3.1.0504 software. The enantiomeric excess $(e e)$ values were calculated from relative peak areas under analytical conditions.

Specific rotations of enantiomers were measured at $589 \mathrm{~nm}$ with a Perkin-Elmer polarimeter model 241 equipped with a Na lamp. The volume of the cell was $2 \mathrm{~mL}$ and the optical path was $10 \mathrm{~cm}$. A standard solution was prepared by dissolving $20 \mathrm{mg}$ of the compounds into $2 \mathrm{~mL}$ of $\mathrm{CHCl}_{3}(c=1)$. The system was set at a temperature of $20^{\circ} \mathrm{C}$ using a Neslab RTE 740 cryostat.

\section{Synthesis}

General procedure for preparation of racemate $( \pm)$-2 and nonchiral analogue 6. A mixture of the appropriate compound $( \pm)-1$ or $5^{15}(7.41 \mathrm{mmol}), \mathrm{K}_{2} \mathrm{CO}_{3}(14.82 \mathrm{mmol})$, and ethyl bromoacetate (11.12 mmol) in $\mathrm{CH}_{3} \mathrm{CN}$ (5 mL) was refluxed under stirring for $2-3 \mathrm{~h}$. The mixture was then concentrated in vacuo, diluted with cold water, and extracted with $\mathrm{CH}_{2} \mathrm{Cl}_{2}(3 \times 15 \mathrm{~mL})$. The organic layer was evaporated in vacuo, and the final compounds $( \pm)-2$ and $6^{16}$ were purified by column chromatography using cyclohexane/ethyl acetate 1:1 as eluent.

( \pm )-ethyl-2-[5-methyl-6-oxo-3-phenyl-5,6-dihydropyridazin-1(4H)yl]acetate $\left[( \pm)\right.$-2]. Yield $=99.9 \%$, yellow oil. ${ }^{1} \mathrm{H} \mathrm{nmR}\left(\mathrm{CDCl}_{3}\right) \delta 1.28$ $\left(\mathrm{m}, 6 \mathrm{H}, \quad \mathrm{CHCH}_{3}+\mathrm{CH}_{2} \mathrm{CH}_{3}\right), 2.67-2.79\left(\mathrm{~m}, 2 \mathrm{H}, \mathrm{CHCH}_{2}\right), \quad 3.07-3.14$ (m, $\left.1 \mathrm{H}, \mathrm{CH}_{3} \mathrm{CH}\right), 4.24\left(\mathrm{q}, 2 \mathrm{H}, \mathrm{CH}_{3} \mathrm{CH}_{2}, \mathrm{~J}=6.9 \mathrm{~Hz}\right), 4.59\left(\mathrm{~s}, 2 \mathrm{H}, \mathrm{NCH}_{2}\right)$, 7.40-7.43 (m, 3H, Ar), 7.72-7.75 (m, 2H, Ar).

General procedure for preparation of racemate $( \pm)-3$ and nonchiral analogue 7. A suspension of the appropriate compound $( \pm)-2$ or $6(7.29 \mathrm{mmol})$ in $6 \mathrm{~N} \mathrm{NaOH}(10 \mathrm{~mL})$ was stirred at $80^{\circ} \mathrm{C}$ for $3-5 \mathrm{~h}$. The mixture was then diluted with cold water and acidified with $6 \mathrm{~N} \mathrm{HCl}$. Products $( \pm)-\mathbf{3}$ and $\mathbf{7}$ were filtered off by suction and recrystallized from ethanol.

( \pm )-2-[5-Methyl-6-oxo-3-phenyl-5,6-dihydropyridazin-1(4H)-yl]acetic acid [( \pm )-3]. Yield $=99.9 \%, \mathrm{mp}=87-89^{\circ} \mathrm{C}(\mathrm{EtOH}) .{ }^{1} \mathrm{H} \mathrm{nmR}\left(\mathrm{CDCl}_{3}\right) \delta 1.31$ (d, $3 \mathrm{H}, \mathrm{CHCH}_{3}, J=6.4 \mathrm{~Hz}$ ), 2.68-2.78 (m, 2H, $\mathrm{CHCH}_{2}$ ), 3.04-3.16 (m, $1 \mathrm{H}, \mathrm{CH}_{3} \mathrm{CH}$ ), $4.64\left(\mathrm{~s}, 2 \mathrm{H}, \mathrm{NCH}_{2}\right), 6.98$ (exch br s, $\left.1 \mathrm{H}, \mathrm{OH}\right), 7.41-7.43$ (m, 3H, Ar), 7.73-7.75 (m, 2H, Ar).

2-[5-Methyl-6-oxo-3-phenylpyridazin-1(6H)-yl]acetic acid (7). Yield $=90 \%, \mathrm{mp}=92-94^{\circ} \mathrm{C}(\mathrm{EtOH}) .{ }^{1} \mathrm{H} \mathrm{nmR}\left(\mathrm{CDCl}_{3}\right) \delta 2.34\left(\mathrm{~s}, 3 \mathrm{H}, \mathrm{CH}_{3}\right)$, 3.48 (exch br s, $1 \mathrm{H}, \mathrm{OH}$ ), 5.05 (s, 2H, $\mathrm{NCH}_{2}$ ), 7.45-7.50 (m, 3H, Ar), 7.63 (s, $1 \mathrm{H}, \mathrm{Ar}), 7.78$ (d, 2H, Ar, J=4.5 Hz).

General procedure for preparation of racemates $( \pm$ )-4a-c and nonchiral analogues 8a-c. To a cooled $\left(-5^{\circ} \mathrm{C}\right)$ and stirred solution of the appropriate derivative $( \pm)-\mathbf{3}$ or $\mathbf{7}(2.06 \mathrm{mmol})$ in anhydrous THF $(6 \mathrm{~mL}), \mathrm{Et}_{3} \mathrm{~N}$ (7.21 mmol) was added. After $30 \mathrm{~min}$, the mixture was allowed to warm up to $0^{\circ} \mathrm{C}$, and ethyl chloroformate $(2.27 \mathrm{mmol})$ was added. After $1 \mathrm{~h}$, the appropriate arylamine $(4.12 \mathrm{mmol})$ was added. The reaction was carried out at room temperature for $12 \mathrm{~h}$. The mixture was then concentrated in vacuo, diluted with cold water $(20-30 \mathrm{~mL})$, and extracted with $\mathrm{CH}_{2} \mathrm{Cl}_{2}(3 \times 15 \mathrm{~mL})$. The solvent was evaporated to obtain the final compounds, which were purified by column chromatography using cyclohexane/ethyl acetate 2:1 (for compound ( \pm )-4a), nhexane/ethyl acetate 3:2 (for compounds $( \pm$ )-4b,c), and cyclohexane/ ethyl acetate 1:1 (for compounds $8 \mathrm{a}-\mathrm{c}$ ) as eluents.

( \pm )-N-(4-Bromophenyl)-2-[5-methyl-6-oxo-3-phenyl-5,6-dihydropyridazin1(4H)-yl]acetamide [( \pm )-4a]. Yield $=23 \%, \mathrm{mp}=148-149^{\circ} \mathrm{C}(\mathrm{EtOH}) .{ }^{1} \mathrm{H}$ $\mathrm{nmR}\left(\mathrm{CDCl}_{3}\right) \delta 1.37(\mathrm{~d}, 3 \mathrm{H}, \mathrm{CHCH}, J=6.4 \mathrm{~Hz}), 2.74-2.81\left(\mathrm{~m}, 2 \mathrm{H}, \mathrm{CHCH}_{2}\right)$, 3.14-3.22 (m, $\left.1 \mathrm{H}, \mathrm{CH}_{3} \mathrm{CH}\right), 4.68\left(\mathrm{~s}, 2 \mathrm{H}, \mathrm{NCH}_{2}\right), 7.23(\mathrm{~d}, 2 \mathrm{H}, \mathrm{Ar}, J=8.8 \mathrm{~Hz})$, 7.38-7.42 (m, 3H, Ar), 7.57 (d, $2 \mathrm{H}, \mathrm{Ar}, J=8.8 \mathrm{~Hz}$ ), 7.77-7.79 (m, 2H, Ar), 8.15 (exch br s, $1 \mathrm{H}, \mathrm{NH}$ ). MS (ESI) calcd. for $\mathrm{C}_{19} \mathrm{H}_{18} \mathrm{BrN}_{3} \mathrm{O}_{2}$, averaged 400.27. Found: $m / z 400 / 402$ with a correct isotopic ratio $1: 1$ of ions species $[\mathrm{M}+\mathrm{H}]^{+}$. Anal. calcd. for $\mathrm{C}_{19} \mathrm{H}_{18} \mathrm{BrN}_{3} \mathrm{O}_{2}: \mathrm{C}, 57.01 ; \mathrm{H}, 4.53 ; \mathrm{N}, 10.50$. Found: C, $56.95 ; \mathrm{H}, 4.51 ; \mathrm{N}, 10.47$.

( \pm )-N-(4-Fluorophenyl)-2-[5-methyl-6-oxo-3-phenyl-5,6-dihydropyridazin1(4H)-yl]acetamide [( \pm -4b]. Yield $=61 \%, \mathrm{mp}=164-165^{\circ} \mathrm{C} \quad(\mathrm{EtOH})$. ${ }^{1} \mathrm{nmR}\left(\mathrm{CDCl}_{3}\right) \delta 1,35\left(\mathrm{~d}, 3 \mathrm{H}, \mathrm{CHCH}{ }_{3}, J=6.4 \mathrm{~Hz}\right), 2,69-2.80\left(\mathrm{~m}, 2 \mathrm{H}, \mathrm{CHCH}_{2}\right)$, 3,11-3,20 (m, $\left.1 \mathrm{H}, \mathrm{CH}_{3} \mathrm{CH}\right), 4,68$ (s, $2 \mathrm{H}, \mathrm{NCH}_{2}$ ), 6.98 (t, $2 \mathrm{H}, \mathrm{Ar}, J=8.8 \mathrm{~Hz}$ ), 7,42-7.49 (m, 5H, Ar), 7,76-7.78 (m, 2H, Ar), 8.25 (exch br s, 1H, NH). MS (ESI) calcd. for $\mathrm{C}_{19} \mathrm{H}_{18} \mathrm{FN}_{3} \mathrm{O}_{2}, 339.36$. Found: $m / z 340[\mathrm{M}+\mathrm{H}]^{+}$. Anal. calcd. for $\mathrm{C}_{19} \mathrm{H}_{18} \mathrm{FN}_{3} \mathrm{O}_{2}$ : C, 67.24; $\mathrm{H}, 5.35 ; \mathrm{N}, 12.38$. Found: $\mathrm{C}, 67.17$; $\mathrm{H}, 5.36$; N, 12.33 .

( \pm )- $N$-(1,3-Benzo-dioxol-5-yl)-2-[5-methyl-6-oxo-3-phenyl-5, 6dihydropyridazin-1 $(\mathbf{4 H})$-yl] acetamide $[( \pm)-4 \mathrm{c}]$.. Yield $=88 \%, \mathrm{mp}=$ $170-171^{\circ} \mathrm{C}(\mathrm{EtOH}) .{ }^{1} \mathrm{H} \mathrm{nmR}\left(\mathrm{CDCl}_{3}\right) \delta 1.35\left(\mathrm{~d}, 3 \mathrm{H}, \mathrm{CHCH}_{3}, J=6.4 \mathrm{~Hz}\right)$, 2.69-2.80 (m, 2H, $\left.\quad \mathrm{CHCH}_{2}\right), \quad 3.10-3.18 \quad\left(\mathrm{~m}, \quad 1 \mathrm{H}, \quad \mathrm{CH}_{3} \mathrm{CH}\right), \quad 4.66$ Chirality DOI 10.1002/chir 
(s, 2H, $\left.\mathrm{NCH}_{2}\right), 5.94\left(\mathrm{~s}, 2 \mathrm{H}, \mathrm{OCH}_{2} \mathrm{O}\right), 6.72(\mathrm{~d}, 1 \mathrm{H}, \mathrm{Ar}, J=8.3 \mathrm{~Hz}), 6.80(\mathrm{dd}, 1 \mathrm{H}$, $\mathrm{Ar}, J=6.2 \mathrm{~Hz}, J=2.1 \mathrm{~Hz}), 7.24(\mathrm{~s}, 1 \mathrm{H}, \mathrm{Ar}), 7.44(\mathrm{t}, 3 \mathrm{H}, \mathrm{Ar}, J=2.8 \mathrm{~Hz}), 7.77-7.79$ (m, $2 \mathrm{H}, \mathrm{Ar}$ ), 8.01 (exch br s, $1 \mathrm{H}, \mathrm{NH}$ ). MS (ESI) calcd. for $\mathrm{C}_{20} \mathrm{H}_{19} \mathrm{~N}_{3} \mathrm{O}_{4}$, 365.38. Found: $m / z 366[\mathrm{M}+\mathrm{H}]^{+}$. Anal. calcd. for $\mathrm{C}_{20} \mathrm{H}_{19} \mathrm{~N}_{3} \mathrm{O}_{4}: \mathrm{C}, 65.74 ; \mathrm{H}$, 5.24 ; N, 11.50. Found: C, 65.61; H, 5.22; N, 11.56 .

$\mathrm{N}$-(4-Bromophenyl)-2-[5-methyl-6-oxo-3-phenylpyridazin-1(6H)yl]acetamide (8a). Yield $=38 \%, \mathrm{mp}=149-151^{\circ} \mathrm{C} \quad(\mathrm{EtOH}) .{ }^{1} \mathrm{H} \quad \mathrm{nmR}$ $\left(\mathrm{CDCl}_{3}\right) \delta 2.37\left(\mathrm{~s}, 3 \mathrm{H}, \mathrm{CH}_{3}\right), 5.08\left(\mathrm{~s}, 2 \mathrm{H}, \mathrm{CH}_{2} \mathrm{CO}\right), 7.40-7.49(\mathrm{~m}, 7 \mathrm{H}$, Ar), 7.68 (s, 1H, Ar), 7.82 (dd, 2H, Ar, J=5.8 Hz, J=1.4 Hz), 9.02 (exch br s, $1 \mathrm{H}, \mathrm{NH}$ ). MS (ESI) calcd. for $\mathrm{C}_{19} \mathrm{H}_{16} \mathrm{BrN}_{3} \mathrm{O}_{2}$, averaged 398.25. Found: $m / z 398 / 400$ with a correct isotopic ratio $1: 1$ of ions species $[\mathrm{M}+\mathrm{H}]^{+}$. Anal. calcd. for $\mathrm{C}_{19} \mathrm{H}_{16} \mathrm{BrN}_{3} \mathrm{O}_{2}: \mathrm{C}, 57.30 ; \mathrm{H}, 4.05 ; \mathrm{N}, 10.55$. Found: C, 57.24; H, 4.07; N, 10.52 .

$\mathrm{N}$-(4-Fluoro phenyl)-2-[5-methyl-6-oxo-3-phenylpyridazin-1(6H)yl]acetamide $\quad(8 b)$. Yield $=45 \%, \quad \mathrm{mp}=117-119^{\circ} \mathrm{C} \quad(\mathrm{EtOH}) .{ }^{1} \mathrm{H} \quad \mathrm{nmR}$ $\left(\mathrm{CDCl}_{3}\right) \delta 2.34\left(\mathrm{~s}, 3 \mathrm{H}, \mathrm{CH}_{3}\right), 5.11$ (s, $\left.2 \mathrm{H}, \mathrm{CH}_{2} \mathrm{CO}\right), 6.89-6.93$ (td, $2 \mathrm{H}, \mathrm{Ar}$, $J=3.3 \mathrm{~Hz}, J=2.0 \mathrm{~Hz}$ ), 7.44-7.49 (m, 5H, Ar), 7.65 (s, $1 \mathrm{H}, \mathrm{Ar}$ ), 7.80 (dd, $2 \mathrm{H}, \mathrm{Ar}, J=3.3 \mathrm{~Hz}, J=2.0 \mathrm{~Hz}$ ), 9.21 (exch br s, $1 \mathrm{H}, \mathrm{NH}$ ). MS (ESI) calcd. for $\mathrm{C}_{19} \mathrm{H}_{16} \mathrm{FN}_{3} \mathrm{O}_{2}$, 337.35. Found: $m / z 338[\mathrm{M}+\mathrm{H}]^{+}$. Anal. calcd. for $\mathrm{C}_{19} \mathrm{H}_{16} \mathrm{FN}_{3} \mathrm{O}_{2}$ : C, 67.65; H, 4.78; N, 12.46. Found: C, 67.72; H, 4.80; N, 12.50 .

$\mathrm{N}$-(1,3-Benzodioxol -5-yl)-2-[5-methyl-6-oxo-3-phenylpyridazin-1 (6H)-yl]acetamide (8c). Yield $=25 \%, \mathrm{mp}=215-217^{\circ} \mathrm{C}(\mathrm{EtOH}) .{ }^{1} \mathrm{H} \mathrm{nmR}$ (DMSO-d $\left.)_{6}\right) \delta 2.19\left(\mathrm{~s}, 3 \mathrm{H}, \mathrm{CH}_{3}\right), 4.95\left(\mathrm{~s}, 2 \mathrm{H}, \mathrm{OCH}_{2} \mathrm{O}\right), 5.99(\mathrm{~s}, 2 \mathrm{H}$, $\mathrm{CH}_{2} \mathrm{CO}$ ), 6.87 (d, $\left.1 \mathrm{H}, \mathrm{Ar}, J=8.4 \mathrm{~Hz}\right), 6.96(\mathrm{~d}, 1 \mathrm{H}, \mathrm{Ar}, J=8.4 \mathrm{~Hz}), 7.29$ (s, 1H, Ar), 7.49 (q, 3H, Ar, J=7.3 Hz), 7.89 (d, 2H, Ar, J=7.3 Hz), 8.04 (s, $1 \mathrm{H}, \mathrm{Ar}$ ), 10.26 (exch br s, $1 \mathrm{H}, \mathrm{NH}$ ). MS (ESI) calcd. for $\mathrm{C}_{20} \mathrm{H}_{17} \mathrm{~N}_{3} \mathrm{O}_{4}$, 363.37. Found: $m / z \quad 364[\mathrm{M}+\mathrm{H}]^{+}$. Anal. calcd for $\mathrm{C}_{19} \mathrm{H}_{16} \mathrm{FN}_{3} \mathrm{O}_{2}$ : C, 66.11; H, 4.72; N, 11.56. Found: C, 66.04; H, 4.70; N, 11.58.

\section{Chiral Chromatographic Resolution}

Analytical chiral HPLC separations of racemates ( \pm )-4a-c were performed by using Lux Amylose-2, Lux Cellulose-1, Lux Cellulose-2 and Lux Cellulose-3 columns ( $50 \mathrm{~mm} \times 4.6 \mathrm{~mm}$ I.D., $3 \mu \mathrm{m}$ ) from Phenomenex (Bologna, Italy) having amylose tris(5-chloro-2-methylphenylcarbamate), cellulose tris(3,5-dimethylphenylcarbamate), cellulose tris(3-chloro-4methylphenylcarbamate), and cellulose tris(4-methylbenzoate) as chiral stationary phases respectively. All chromatographic resolutions were performed at $40^{\circ} \mathrm{C}$ using isocratic elutionand alternatively mixtures of $\mathrm{MeCN}$ or $n$-Hex and different concentrations of IPA as the polar modifier. The dead times $\left(\mathrm{t}_{0}\right)$ of the tested columns, estimated at flow rate of $1.0 \mathrm{~mL} / \mathrm{min}$, were comparable in the range of $0.7-0.8 \mathrm{~min}$. The values of $t_{0}$ found for each column and for each mobile phase employed were used for the retention factor $\left(\mathrm{k}^{\prime}\right)$ determination. The $\mathrm{k}^{\prime}$ was calculated using the equation $k^{\prime}=\left(t_{r}-t_{0}\right) / t_{0}$, where $t_{r}$ is the retention time. The enantioselectivity $(\alpha)$ and the resolution factors $(\mathrm{R})$ were calculated as follows: $\alpha=k_{2}{ }^{\prime} / k_{1}{ }^{\prime}=2\left(t_{r, 2}-t_{r, 1}\right) /\left(w_{1}+w_{2}\right)$, where $t_{r, 2}$ and $t_{r, 1}$ are the retention times of the second and the first enantiomers respectively, while $\mathrm{w}_{1}$ and $\mathrm{w}_{2}$ are the corresponding base peak widths. Table 1 reports the analytical parameters for the resolution of racemates $( \pm$ )-4a-c carried out on the four amylose- and cellulose-derived CSPs, using mixtures of MeCN or $n$-Hex containing $10 \%$ IPA.

From analysis of the data obtained in analytical enantioseparations (Table 1), we found the best chromatographic profile for the resolution of all racemic mixtures $( \pm)-4 \mathrm{a}-\mathrm{c}$ was achieved with a Lux Amylose-2 column using MeCN/IPA 90:10 as the mobile phase (Fig. 2). These conditions were then chosen for semipreparative purification, which was found in analytical experiments to represent the best compromise between high resolution of the two enantiomers, high loading capacity of analytes, and short separation time. Quantitative isolation of the optically active isomers (-)-4a-c and (+)-4a-c was then performed at $40^{\circ} \mathrm{C}$ on a semipreparative apparatus, using a Lux Amylose-2 column $(250 \mathrm{~mm} \times 4.6 \mathrm{~mm}$ I.D., $5 \mu \mathrm{m})$ and $\mathrm{MeCN} / \mathrm{IPA}$ 90:10 as the isocratic mobile phase. The flow rate was $1 \mathrm{~mL} / \mathrm{min}$ and the sample injection was in the range of 150 to $200 \mu \mathrm{g}$ of analyte for each run. Wavelength used for the detection was $250 \mathrm{~nm}$. Finally, pure enantiomers were properly recovered by partition of the eluate according to the profile of the chromatograms, as shown in Figure 3.

$S$-(-)-N-(4-Bromophenyl)-2-[5-methyl-6-oxo-3-phenyl-5,6dihydropyridazin-1(4H)-yl] acetamide [S(-)-4a]. $\mathrm{mp}=148-149^{\circ} \mathrm{C}$ (EtOH). $[\alpha]^{20}{ }_{\mathrm{D}}=-117.8^{\circ}$ (c 1, $\left.\mathrm{CHCl}_{3}\right) . e e=99.9 \%{ }^{1} \mathrm{H} \mathrm{nmR}\left(\mathrm{CDCl}_{3}\right) \delta 1.33$ (d, $3 \mathrm{H}, \mathrm{CH}_{2} \mathrm{CHCH}_{3}, \mathrm{~J}=6.4 \mathrm{~Hz}$ ), 2.69-2.79 (m, 2H, $\mathrm{CH}_{2} \mathrm{CHCH}_{3}$ ), 3.11-3.19 (m, $1 \mathrm{H}, \mathrm{CH}_{2} \mathrm{CHCH}_{3}$ ), 4.64 (s, $2 \mathrm{H}, \mathrm{NCH}_{2} \mathrm{CO}$ ), 7.40-7.43 (m, 7H, Ar), 7.747.77 (m, 2H, Ar), 8.07 (exch br s, 1H, NH). MS (ESI) calcd. for $\mathrm{C}_{19} \mathrm{H}_{18} \mathrm{BrN}_{3} \mathrm{O}_{2}$, averaged 400.27. Found: $m / z$ 400/402 with a correct isotopic ratio $1: 1$ of ions species $[\mathrm{M}+\mathrm{H}]^{+}$. Anal. calcd. for $\mathrm{C}_{19} \mathrm{H}_{18} \mathrm{BrN}_{3} \mathrm{O}_{2}$ : C, 57.01; H, 4.53; N, 10.50. Found: C, 57.07; H, 4.55; N, 10.53 .

$R$-(+)-N-(4-Bromophenyl)-2-[5-methyl-6-oxo-3-phenyl-5,6dihydropyridazin-1(4H)-yl] acetamide [R-(+)-4a]. $\mathrm{mp}=148-149^{\circ} \mathrm{C}$ (EtOH). $[\alpha]^{20}{ }_{\mathrm{D}}=+114.2^{\circ}\left(c 1, \mathrm{CHCl}_{3}\right) \cdot e e=98.1 \%{ }^{1} \mathrm{H} \mathrm{nmR}\left(\mathrm{CDCl}_{3}\right)$ and $\mathrm{MS}$ (ESI) spectra are identical to that of the enantiomer $S$-(-)-4a. Anal. calcd for $\mathrm{C}_{19} \mathrm{H}_{18} \mathrm{BrN}_{3} \mathrm{O}_{2}$ : C, $57.01 ; \mathrm{H}, 4.53 ; \mathrm{N}, 10.50$. Found: C, 57.12; H, 4.52; N, 10.52 .

$S$-(-)-N-(4-Fluorophenyl)-2-[5-methyl-6-oxo-3-phenyl-5,6dihydropyridazin-1(4H)-yl] acetamide [S-(-)-4b]. $\mathrm{mp}=164-165^{\circ} \mathrm{C}$ (EtOH). $[\alpha]^{20}{ }_{\mathrm{D}}=-133.8^{\circ}\left(c 1, \mathrm{CHCl}_{3}\right) . e e=98.1 \% .{ }^{1} \mathrm{H} \mathrm{nmR}\left(\mathrm{CDCl}_{3}\right) \delta 1.34$ (d, $3 \mathrm{H}, \mathrm{CH}_{2} \mathrm{CHCH}_{3}, \mathrm{~J}=6.4 \mathrm{~Hz}$ ), 2.68-2.79 (m, 2H, $\mathrm{CH}_{2} \mathrm{CHCH}_{3}$ ), 3.11-3.19 (m, $1 \mathrm{H}, \mathrm{CH}_{2} \mathrm{CHCH}_{3}$ ), 4.65 (s, $2 \mathrm{H}, \mathrm{NCH}_{2} \mathrm{CO}$ ), 6.98 (t, $2 \mathrm{H}, \mathrm{Ar}, J=8.8 \mathrm{~Hz}$ ),

TABLE 1. Analytical chromatographic parameters of compounds ( \pm )-4a-c calculated on cellulose and amylose CSPs tested

\begin{tabular}{|c|c|c|c|c|c|c|c|c|c|c|c|c|}
\hline \multirow{2}{*}{$\begin{array}{l}\text { Column and } \\
\text { Mobile Phase }\end{array}$} & \multicolumn{4}{|c|}{$( \pm)-4 \mathrm{a}$} & \multicolumn{4}{|c|}{$( \pm)-4 \mathrm{~b}$} & \multicolumn{4}{|c|}{$( \pm)-4 \mathrm{c}$} \\
\hline & $\mathrm{k}_{1}^{\prime}$ & $\mathrm{k}_{2}^{\prime}$ & $\alpha$ & $\mathrm{R}$ & $\mathrm{k}_{1}^{\prime}$ & $\mathrm{k}_{2}^{\prime}$ & $\alpha$ & $\mathrm{R}$ & $\mathrm{k}_{1}^{\prime}$ & $\mathrm{k}_{2}$ & $\alpha$ & $\mathrm{R}$ \\
\hline MeCN:IPA 9:1 & 0.85 & 2.50 & 2.95 & 3.9 & 0.40 & 1.00 & 2.52 & 2.5 & 1.14 & 3.02 & 2.65 & 3.5 \\
\hline $\begin{array}{l}\text { Hex:IPA 9:1 } \\
\text { Lux Cellulose-1 }\end{array}$ & 21.73 & 29.41 & 1.35 & 3.2 & 27.58 & 31.40 & 1.14 & 1.4 & $\mathrm{NR}^{\mathrm{b}}$ & NR & NR & NR \\
\hline MeCN:IPA 9:1 & 0.28 & 0.41 & 1.44 & 0.6 & 0.19 & 0.24 & 1.27 & 0.3 & NR & NR & NR & NR \\
\hline MeCN:IPA 9:1 & NR & NR & NR & NR & NR & NR & NR & NR & NR & NR & NR & NR \\
\hline $\begin{array}{l}\text { Hex:IPA 9:1 } \\
\text { Lux Cellulose-3 }\end{array}$ & 13.07 & 14.01 & 1.07 & 0.5 & 11.87 & 13.33 & 1.12 & 0.6 & NR & NR & NR & NR \\
\hline MeCN:IPA 9:1 & NR & NR & NR & NR & NR & NR & NR & NR & NR & NR & NR & NR \\
\hline Hex:IPA 9:1 & 4.62 & 5.25 & 1.14 & 1.0 & 3.53 & 3.85 & 1.09 & 0.7 & 11.95 & 12.59 & 1.05 & 0.5 \\
\hline
\end{tabular}

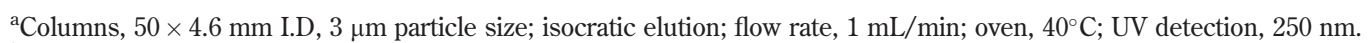

${ }^{b} \mathrm{NR}$, no resolution due to the lack of selectivity or strong retention on the stationary phase.

Chirality DOI 10.1002/chir 

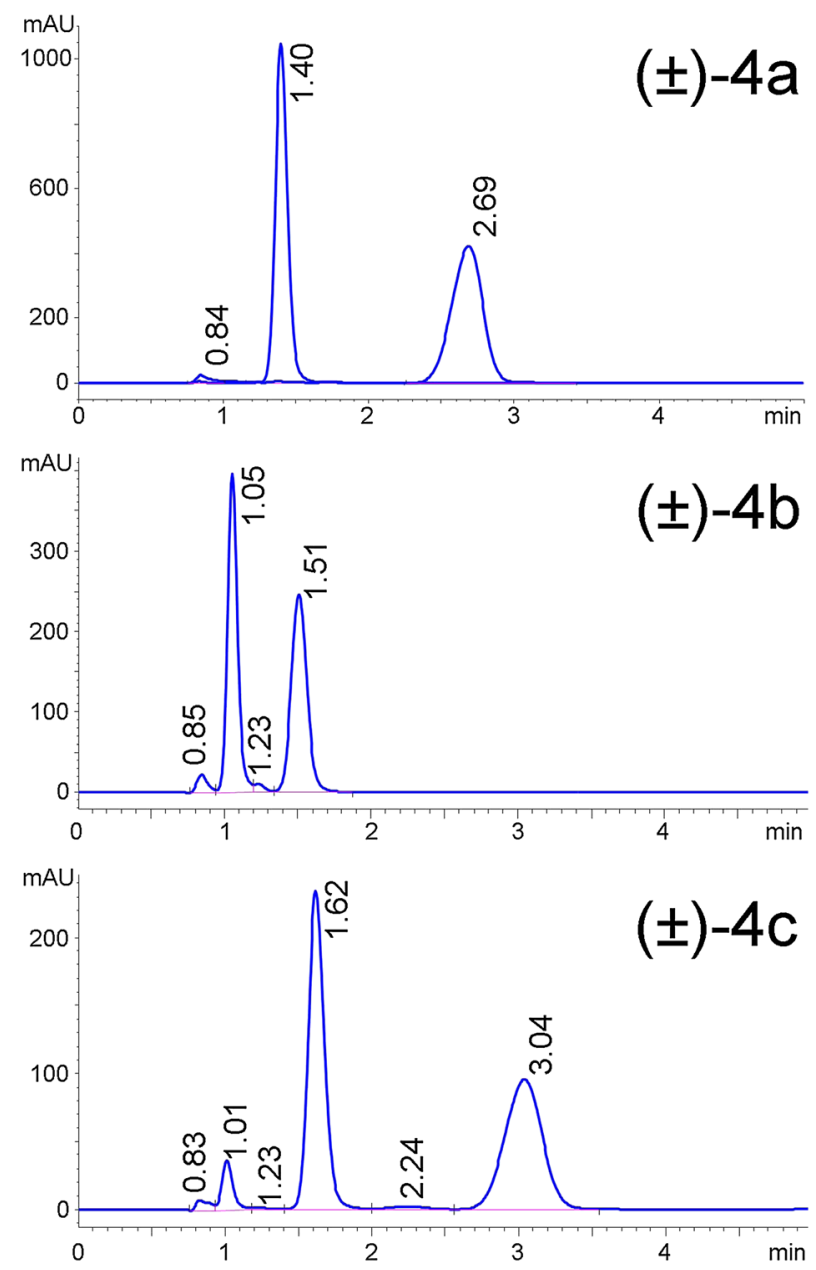

Fig. 2. Analytical separation of racemates $( \pm)-4 a-c$ obtained by simultaneous on-line UV detection. Column, Lux Amylose-2 (50 mm $\times 4.6 \mathrm{~mm}$ I.D., $3 \mu \mathrm{m})$; eluent, MeCN/IPA 90:10; flow rate, $1.0 \mathrm{~mL} / \mathrm{min}$; temperature, $40^{\circ} \mathrm{C}$; detection, $250 \mathrm{~nm}$; for other experimental conditions, see Table 1.

7.41-7.47 (m, 5H, Ar), 7.74-7.80 (m, 2H, Ar), 8.01 (exch br s, $1 \mathrm{H}, \mathrm{NH}$ ). MS (ESI) calcd. for $\mathrm{C}_{19} \mathrm{H}_{18} \mathrm{FN}_{3} \mathrm{O}_{2}, 339.36$. Found: $m / z 340[\mathrm{M}+\mathrm{H}]^{+}$. Anal. calcd. for $\mathrm{C}_{19} \mathrm{H}_{18} \mathrm{FN}_{3} \mathrm{O}_{2}$ : C, 67.24; $\mathrm{H}, 5.35 ; \mathrm{N}, 12.38$. Found: C, 67.31; $\mathrm{H}$, $5.34 ; \mathrm{N}, 12.43$.

$R-(+)-N$-(4-Fluorophenyl)-2-[5-methyl-6-oxo-3-phenyl-5,6dihydropyridazin-1 $(\mathbf{4 H})$-yl]acetamide $[\boldsymbol{R}-(+)-4 \mathrm{~b}] . \mathrm{mp}=164-165^{\circ} \mathrm{C}$ (EtOH). $[\alpha]^{20}{ }_{\mathrm{D}}=+128.3^{\circ}\left(c \quad 1, \mathrm{CHCl}_{3}\right) . e e=99.1 \% .{ }^{1} \mathrm{H} \mathrm{nmR}\left(\mathrm{CDCl}_{3}\right)$ and

\begin{tabular}{|c|c|c|c|c|}
\hline N3A -H3A & N3A . . O1B & H3A ..O1B & N3A -H3A. .O1B & Operation symmetry \\
\hline $0.86(.01)$ & $2.94(.01)$ & $2.10(.01)$ & $164.2(0.6)$ & $\mathrm{x}+1,+\mathrm{y},+\mathrm{z}$ \\
\hline $0.860(0.008)$ & $2.89(0.1)$ & $2.045(0.09)$ & $166.9(0.6)$ & $\mathrm{x},+\mathrm{y}+1,+\mathrm{z}$ \\
\hline $0.860(0.009)$ & $2.87(0.1)$ & $2.013(0.08)$ & $173.6(0.7)$ & $\mathrm{x},+\mathrm{y}-1,+\mathrm{z}$ \\
\hline
\end{tabular}

MS (ESI) spectra are identical to that of the enantiomer $S-(-)-4 \mathrm{~b}$. Anal. calcd for $\mathrm{C}_{19} \mathrm{H}_{18} \mathrm{FN}_{3} \mathrm{O}_{2}$ : C, 67.24; $\mathrm{H}, 5.35 ; \mathrm{N}, 12.38$. Found: C, 67.10; $\mathrm{H}, 5.37 ; \mathrm{N}, 12.40$.

$\mathrm{S}$-(-)-N-(1,3-Benzo-dioxol-5-yl)-2-[5-methyl-6-oxo-3-phenyl-5,6dihydropyridazin-1(4H)-yl] acetamide $[S-(-)-4 c] . \quad m p=170-171^{\circ} \mathrm{C}$ (EtOH). $[\alpha]^{20}{ }_{\mathrm{D}}=-132.3^{\circ}\left(c 1, \mathrm{CHCl}_{3}\right) . e e=99.9 \% .{ }^{1} \mathrm{H} \mathrm{nmR}\left(\mathrm{CDCl}_{3}\right) \delta 1.35$ (d, $3 \mathrm{H}, \mathrm{CH}_{2} \mathrm{CHCH}_{3}, J=6.4 \mathrm{~Hz}$ ), 2.67-2.78 (m, $2 \mathrm{H}, \mathrm{CH}_{2} \mathrm{CHCH}_{3}$ ), 3.10-3.18 (m, $1 \mathrm{H}, \mathrm{CH}_{2} \mathrm{CHCH}_{3}$ ), 4.63 (s, $2 \mathrm{H}, \mathrm{NCH}_{2} \mathrm{CO}$ ), 5.92 (s, $\left.2 \mathrm{H}, \mathrm{OCH}_{2} \mathrm{O}\right), 6.71$ (d, $1 \mathrm{H}, \mathrm{Ar}, J=8.3 \mathrm{~Hz}$ ), 6.80 (dd, $1 \mathrm{H}, \mathrm{Ar}, J=6.4 \mathrm{~Hz}, J=1.9 \mathrm{~Hz}$ ), 7.21 (s, $1 \mathrm{H}$,
Ar), 7.40-7.42 (m, 3H, Ar), 7.74-7.77 (m, 2H, Ar), 7.88 (exch br s, $1 \mathrm{H}$, NH). MS (ESI) calcd. for $\mathrm{C}_{20} \mathrm{H}_{19} \mathrm{~N}_{3} \mathrm{O}_{4}, 365.38$. Found: $m / z 366[\mathrm{M}+\mathrm{H}]^{+}$. Anal. calcd for $\mathrm{C}_{20} \mathrm{H}_{19} \mathrm{~N}_{3} \mathrm{O}_{4}$ : C, 65.74; H, 5.24; N, 11.50. Found: C, 65.81; $\mathrm{H}, 5.26$; N, 11.53 .

$R$-(+)-N-(1,3-Benzo-dioxol-5-yl)-2-[5-methyl-6-oxo-3-phenyl-5,6dihydropyridazin-1(4H)-yl] acetamide $[\boldsymbol{R}-(+)-4 \mathrm{c}] . \mathrm{mp}=170-171^{\circ} \mathrm{C}$ $(\mathrm{EtOH}) \cdot[\alpha]^{20}{ }_{\mathrm{D}}=+136 \cdot 6^{\circ}\left(c 1, \mathrm{CHCl}_{3}\right) . e e=99 \cdot 2 \% .{ }^{1} \mathrm{H} \mathrm{nmR}$ and MS (ESI) spectra are identical to that of the enantiomer $S-(-)-4 \mathrm{c}$. Anal. calcd. for $\mathrm{C}_{20} \mathrm{H}_{19} \mathrm{~N}_{3} \mathrm{O}_{4}$ : C, 65.74; H, 5.24; N, 11.50. Found: C, 65.87; H, 5.23; N, 11.46.

\section{X-ray Crystal Structure Analysis}

X-ray diffraction analysis of compounds $S-(-)-4 a, c$ and $R-(+)-$ 4b. X-ray analyses were carried out with a Goniometer Oxford Diffraction KM4 Xcalibur2. Graphite-monochromated $\mathrm{Mo} / \mathrm{K} \alpha$ radiation $(40 \mathrm{~mA} /-40$ $\mathrm{KV}$ ) and a KM4 CCD/SAPPHIRE detector were used for cell parameter determination and data collection of $S-(-)-4 \mathrm{a}$ and $S-(-)-4 \mathrm{c}$. $\mathrm{Cu} / \mathrm{K} \alpha$ radiation $(40 \mathrm{~mA} /-40 \mathrm{KV})$, monochromated by an Oxford Diffraction Enhance ULTRA assembly, and an Oxford Diffraction Excalibur PX Ultra CCD were used for cell parameter determination and data collection of $R-(+)-4 \mathrm{~b}$. The integrated intensities, measured using the $\omega$ scan mode, were corrected for Lorentz and polarization effects. ${ }^{17}$ The substantial redundancy in data allowed empirical absorption corrections $\left(\mathrm{SADABS}^{18}\right)$ to be applied using multiple measurements of symmetry-equivalent reflections. The structure was solved by direct methods of SIR2 $2004^{19}$ and refined using the fullmatrix least squares on $\mathrm{F}^{2}$ provided by SHELXL97. ${ }^{20}$ In all three compounds, the non-hydrogen atoms were refined anisotropically. Aromatic, methylic, methylenic, and methinic hydrogens were assigned in calculated positions, only hydrogen on N-3 in $S$-(-)-4c was found in the Fourier synthesis; all hydrogen atoms were refined as isotropic.

The X-ray CIF file for each of the three structures has been deposited at the Cambridge Crystallographic Data Center and allocated with the deposition numbers CCDC 833484 [S-(-)-4a], CCDC 833486 [R-(+)-4b] and CCDC $833485[\mathbf{S}(-)-4 \mathbf{c}]$ respectively. Copies of the data can be obtained, free of charge, from CCDC, 12 Union Road, Cambridge, CB2 1EZ UK (e-mail: deposit@ccdc.cam.ac.uk; internet: //www.ccdc. cam.ac.uk).

Compound $\mathbf{S}-(-)-4 a . \quad 4 \mathrm{x}\left(\mathrm{C}_{19} \mathrm{H}_{18} \mathrm{~N}_{3} \mathrm{O}_{2} \mathrm{Br}\right), \mathrm{M}=1601.10$, Triclinic, space group P1, $a=10.070(1), b=12.244(1), c=15.328(1) \AA{ }^{\prime}, \mathrm{V}=1832.0(3) \AA^{3}{ }^{3}$ $\mathrm{Z}=1 \quad \mathrm{D}_{\mathrm{c}}=1.451, \mu=2.261 \mathrm{~mm}^{-1}, \mathrm{~F}(000)=816$, experiment $\mathrm{T}=293^{\circ} \mathrm{K}$. Single crystals of $S$-(-)-4a were obtained by slow crystallization from $\mathrm{EtOH}$. The asymmetric unit contained four independent molecules, as indicated in the molecular formula. 13926 reflections were collected with a $4.27<\theta<29.41$ range; 9827 were independent; the parameters were 902 and the final $\mathrm{R}$ index was 0.0518 for reflections having $\mathrm{I}>2 \sigma \mathrm{I}$. There are four non equivalent molecules in the asymmetric unit. In PARST analysis, we found three significant intermolecular hydrogen bonds. They have the following parameters and show interactions along the a and $b$ axes:

Compound $\boldsymbol{R}$-(+)-4b. $2 \times\left(\mathrm{C}_{19} \mathrm{H}_{18} \mathrm{FN}_{3} \mathrm{O}_{2}\right), \mathrm{M}=678.73$, Tetragonal, space group $\mathrm{P}-421 \mathrm{c}, a=19.982(1), b=19.982(1), c=17.455(1) \AA$, $\mathrm{V}=6969.4(6) \AA{ }^{3} \quad Z=8 \quad \mathrm{D}_{\mathrm{c}}=1.294, \quad \mu=0.768 \quad \mathrm{~mm}^{-1}, \quad \mathrm{~F}(000)=2848$, experiment $\mathrm{T}=293^{\circ} \mathrm{K}$. Single crystals of $R-(+)-4 \mathrm{~b}$ were obtained by slow crystallization from $\mathrm{MeCN}$. The asymmetric unit contains two independent molecules, as indicated in the molecular formula. 48444 reflections were collected with a $4.43<\theta<65.20$ range; 5917 were independent; the parameters were 452 and the final $\mathrm{R}$ index was 0.0682 for reflections having $\mathrm{I}>2 \sigma \mathrm{I}$. In PARST analysis, we found two significant hydrogen bonds. The first is 

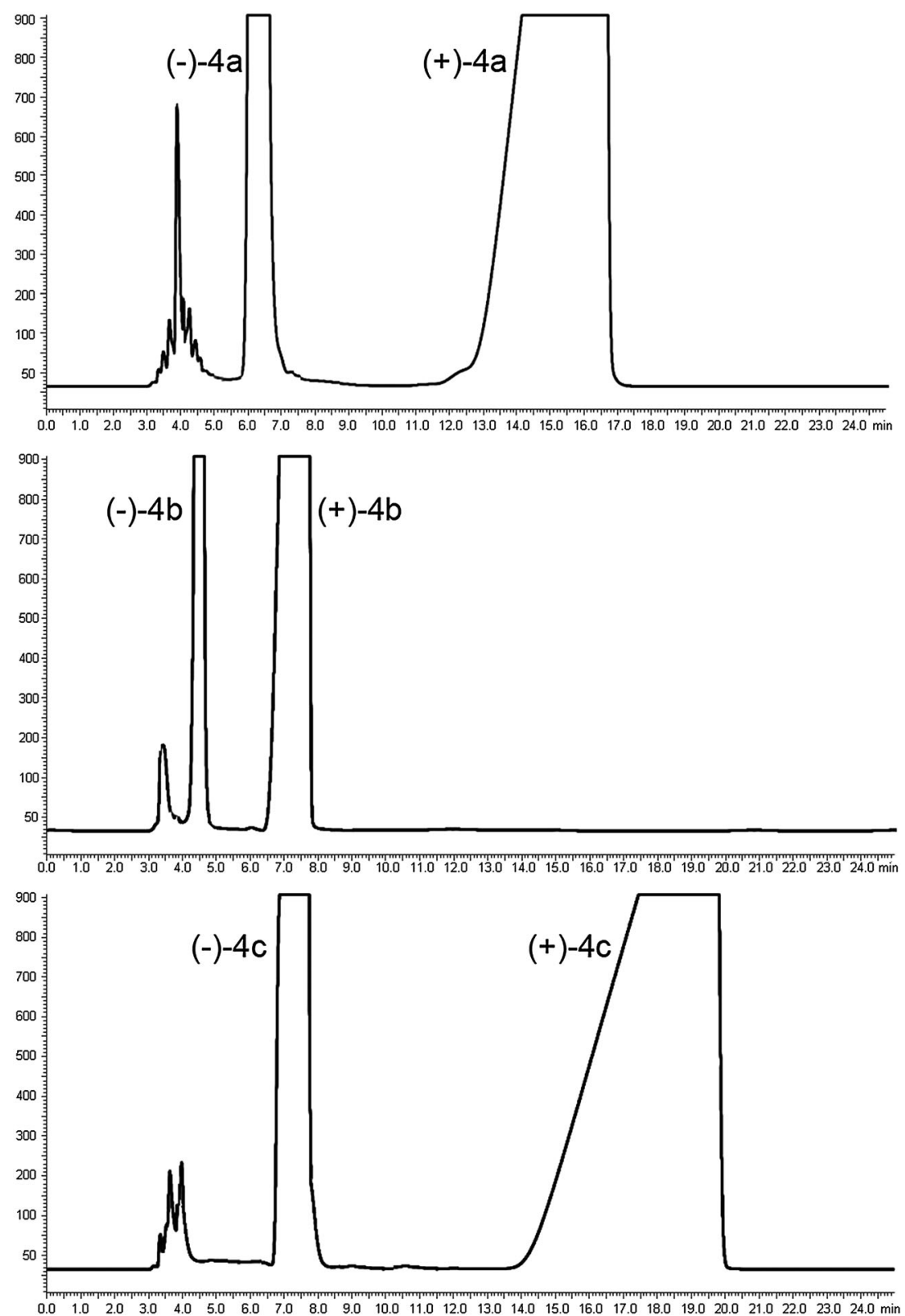

Fig. 3. Semipreparative resolution of racemates ( \pm )-4a-c. Column, Lux Amylose-2 (250 mm × $4.6 \mathrm{~mm}$ I.D., $5 \mu \mathrm{m}$ ); eluent, MeCN/IPA 90:10; flow-rate, 1.0 mL/ min; temperature, $40^{\circ} \mathrm{C}$; detection, $250 \mathrm{~nm}$.

intramolecular, whereas the second is intermolecular. They have the following parameters: analysis, we found a significant intermolecular hydrogen bond that has the following parameters and shows an interaction along the a axis:

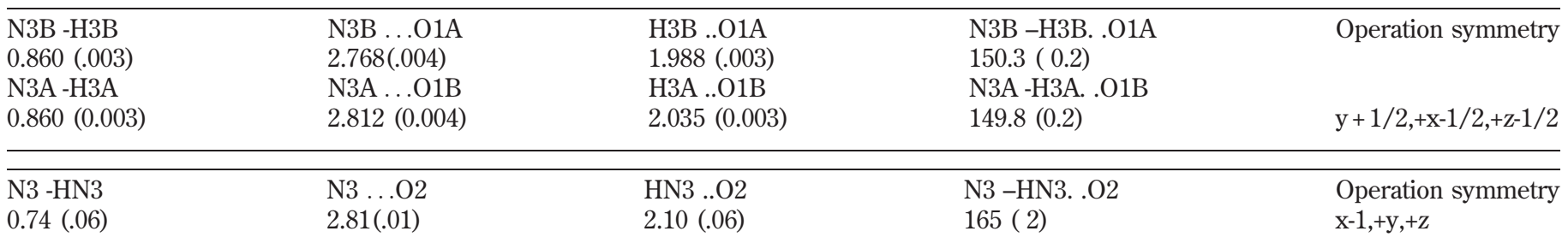

Compound S-(-)-4c. $\mathrm{C}_{20} \mathrm{H}_{19} \mathrm{~N}_{3} \mathrm{O}_{4}, \mathrm{M}=365.38$, Orthorhombic, space group P 2121 21, $a=4.721$ (1), $b=16.863(3), c=21.597(3) \AA$, $\mathrm{V}=1719.3(5)$ $\AA^{3}, \mathrm{Z}=4 \mathrm{D}_{\mathrm{c}}=1.412, \mu=0.100 \mathrm{~mm}^{-1}, \mathrm{~F}(000)=768$, experiment $\mathrm{T}=150^{\circ} \mathrm{K}$. Single crystals of $S-(-)-4 c$ were obtained by vapour diffusion from the $\mathrm{EtOH} / n$-hexane mixture. 5351 reflections were collected with a $4.42<\theta<28.02$ range; 3243 were independent; the parameters were 248 and the final $\mathrm{R}$ index was 0.0981 for reflections having $\mathrm{I}>2 \sigma \mathrm{I}$. In PARST Chirality DOI 10.1002/chir

\section{Biological Assays}

Cell Culture. Human promyelocytic leukemia HL-60 cells stably transfected with FPR1 (HL-60-FPR1), FPR2 (HL-60-FPR2), or FPR3 (HL-60-FPR3) were cultured in RPMI 1640 medium supplemented with $10 \%$ heat-inactivated fetal calf serum, $10 \mathrm{~mm}$ HEPES, $100 \mu \mathrm{g} / \mathrm{ml}$ streptomycin, $100 \mathrm{U} / \mathrm{ml}$ penicillin, and G418 $(1 \mathrm{mg} / \mathrm{mL})$, as previously 
described. ${ }^{21}$ Wild-type HL-60 cells were cultured under the same conditions, but without G418.

Isolation of Human Neutrophils. Blood was collected from healthy donors in accordance with a protocol approved by the Institutional Review Board at Montana State University. Neutrophils were purified from the blood using dextran sedimentation, followed by Histopaque 1077 gradient separation and hypotonic lysis of red blood cells, as previously described. ${ }^{22}$ Isolated neutrophils were washed twice and resuspended in Hank's balanced-salt solution without $\mathrm{Ca}^{2+}$ and $\mathrm{Mg}^{2+}$ (HBSS). Neutrophil preparations were routinely $>95 \%$ pure, as determined by light microscopy, and $>98 \%$ viable, as determined by trypan blue exclusion.

$\mathrm{Ca}^{2+}$ Mobilization Assay. Changes in intracellular $\mathrm{Ca}^{2+}$ were measured with a FlexStation II scanning fluorometer using a FLIPR 3 calcium assay kit (Molecular Devices, Sunnyvale, CA, USA) for human neutrophils and HL-60 cells. All active compounds were evaluated in parent (wild-type) HL-60 cells for supporting that the agonists are inactive in non-transfected cells. Human neutrophils or HL-60 cells, suspended in HBSS containing $10 \mathrm{~mm}$ HEPES, were loaded with Fluo-4 AM dye (Invitrogen) $(1.25 \mu \mathrm{g} / \mathrm{mL}$ final concentration) and incubated for $30 \mathrm{~min}$ in the dark at $37^{\circ} \mathrm{C}$. After dye loading, the cells were washed with HBSS containing $10 \mathrm{~mm}$ HEPES, resuspended in HBSS containing $10 \mathrm{~mm}$ HEPES and $\mathrm{Ca}^{2+}$ and $\mathrm{Mg}^{2+}$ (HBSS ${ }^{+}$, and aliquotted into the wells of a flat-bottomed, half-area-well black microtiter plates $\left(2 \times 10^{5}\right.$ cells/well). The compound source plate contained dilutions of test compounds in HBSS $^{+}$. Changes in fluorescence were monitored $\left(\lambda_{\text {ex }}=485 \mathrm{~nm}, \lambda_{\text {em }}=538\right.$ $\mathrm{nm}$ ) every $5 \mathrm{sec}$ for $240 \mathrm{sec}$ at room temperature after automated addition of compounds. Maximum change in fluorescence, expressed in arbitrary units over baseline, was used to determine agonist response. Responses were normalized to the response induced by $5 \mathrm{~nm}$ fMLF (Sigma Chemical Co., St. Louis, MO, USA) for HL-60-FPR1 and neutrophils, or $5 \mathrm{~nm}$ WKYMVm (Calbiochem, San Diego, CA, USA) for HL-60-FPR2 and HL60 -FPR3 cells, which were assigned a value of $100 \%$. Curve fitting (5-6 points) and calculation of median effective concentration values $\left(\mathrm{EC}_{50}\right)$ were performed by nonlinear regression analysis of the dose-response curves generated using Prism 5 (GraphPad Software, Inc., San Diego, CA, USA).

\section{RESULTS AND DISCUSSION Synthesis}

The already described $( \pm)-1$ and aromatic analogue $5,{ }^{15}$ which was obtained from $( \pm)-1$ with bromine in acetic acid as the oxidizing agent ${ }^{23}$ (Scheme 1), were the key building blocks for synthesis of the final compounds $( \pm)-4 a-c$ and $8 \mathrm{a}-\mathrm{c}$. The alkylation under standard conditions with ethyl bromoacetate resulted in the racemate $( \pm)-2$ and the known non-chiral $6,{ }^{16}$ from which the corresponding intermediates $( \pm)-3$ and 7 were obtained after alkaline hydrolysis. The final racemates $( \pm)-4 \mathrm{a}-\mathrm{c}$ and the non-chiral analogues $8 \mathrm{a}-\mathrm{c}$ were prepared in good yields by treating the corresponding carboxylic acids ( \pm -3 and 7 with ethyl chloroformate in THF in the presence of triethylamine; the intermediate mixed anhydrides were not isolated but were directly transformed into the final amides using the appropriate aryl amine (Scheme 1). The pure compounds $( \pm)-4 \mathrm{a}-\mathrm{c}$ and $8 \mathrm{a}-\mathrm{c}$ were crystallized from ethanol, and the results of ${ }^{1} \mathrm{H}$ nmR, MS(ESI), and elemental analysis agreed with the assigned structure.

\section{Chiral Resolution}

In order to obtain complete resolution of the racemates $( \pm)$ 4a-c, we performed an analytical chromatographic approach based on the screening of four different chiral stationary phases: Lux Amylose-2, Lux Cellulose-1, Lux Cellulose-2 and Lux Cellulose-3 (50 $\mathrm{mm} \times 4.6 \mathrm{~mm}$ I.D., $3 \mu \mathrm{m}$ particle size). Different mobile phases were tested to determine the best combination of mobile and stationary phases for the resolution of racemates. The chromatographic results of the screening are reported in Table 1 as retention factor ( $\left.k^{\prime}\right)$, enantioselectivity $(\alpha)$ and resolution factors (R). Experimental data indicated that

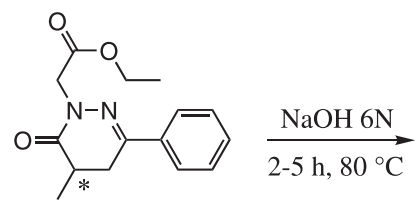

$( \pm)-2$

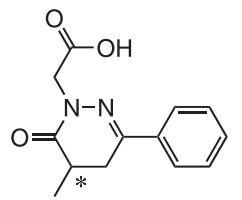

$( \pm)-3$

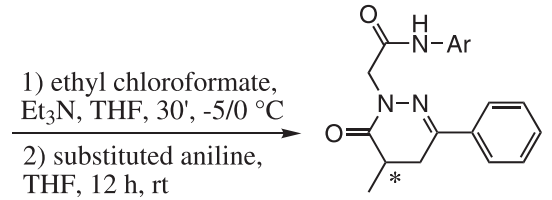

$( \pm)-4 a-c$

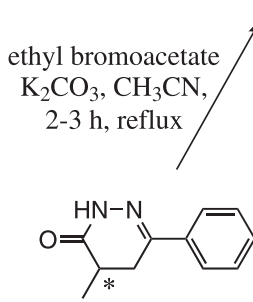

$( \pm)-1$
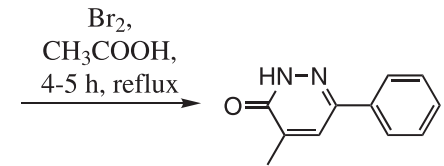

5

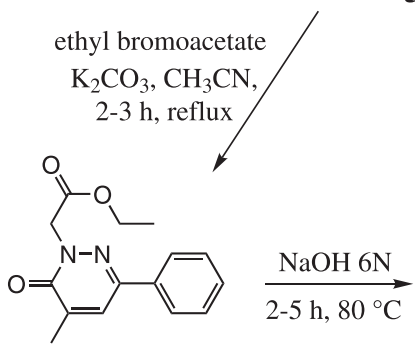

6

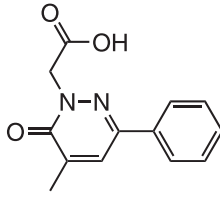

7

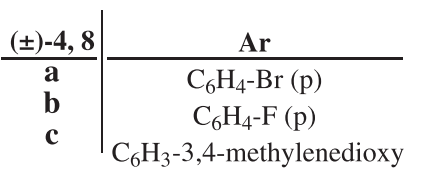

1) ethyl chloroformate, $\mathrm{Et}_{3} \mathrm{~N}, \mathrm{THF}, 30^{\prime},-5 / 0^{\circ} \mathrm{C}$

2) substituted aniline, THF, $12 \mathrm{~h}, \mathrm{rt}$

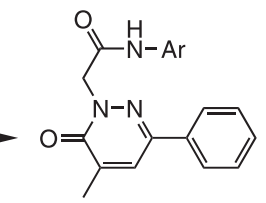

8a-c

Scheme 1. Synthesis of chiral compounds $( \pm)-4 a-c$ and non-chiral analogues 8 a c. 

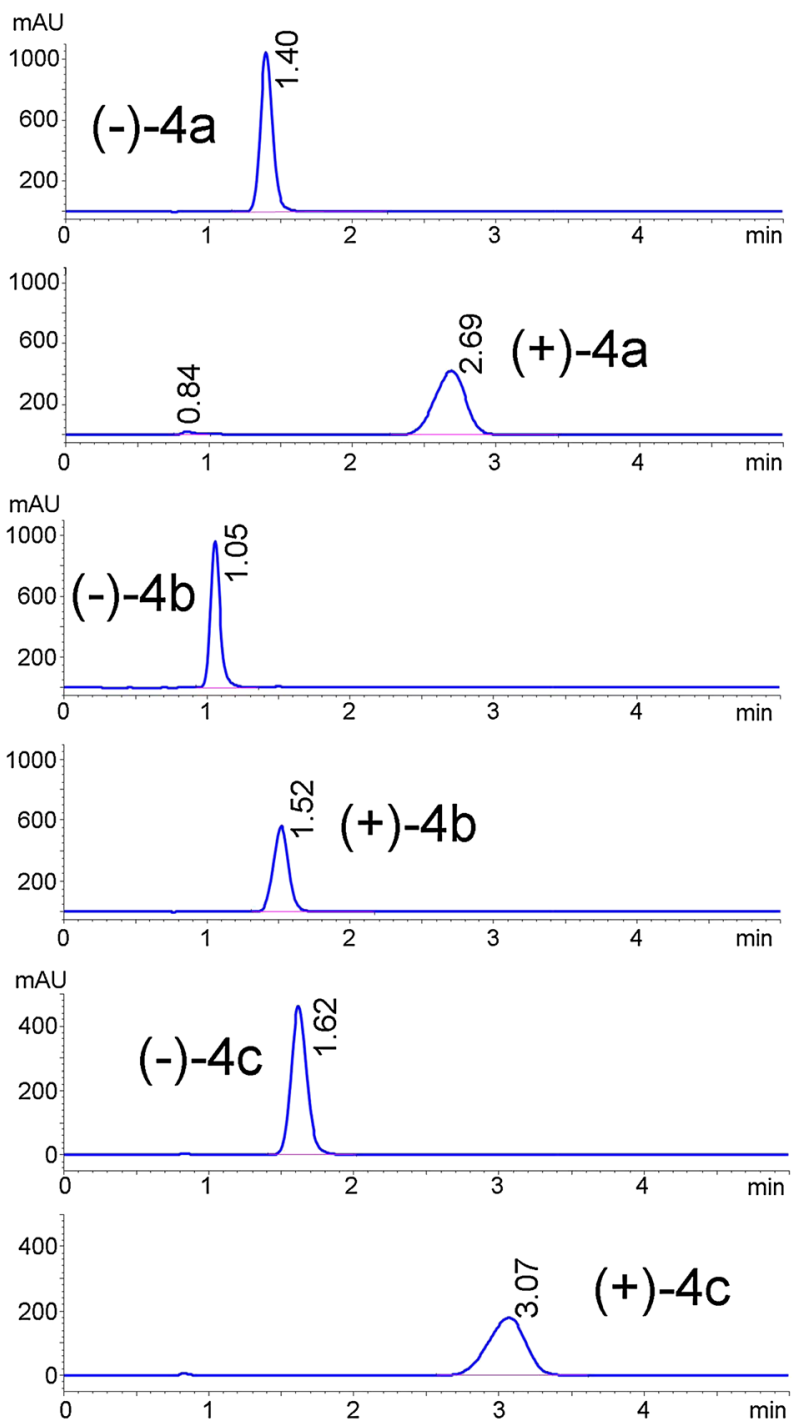

Fig. 4. Chiral chromatographic analysis of isolated enantiomers (-)-4a-c and (+)-4a-c to determine enantiomeric excess (ee). Column, Lux Amylose-2 $(50 \mathrm{~mm} \times 4.6 \mathrm{~mm}$ I.D., $3 \mu \mathrm{m})$; eluent, MeCN/IPA 90:10; flow rate, $1.0 \mathrm{~mL} / \mathrm{min}$; temperature, $40^{\circ} \mathrm{C}$; detection, $250 \mathrm{~nm}$.

the best resolutions $(\mathrm{R}>1.5)$ were achieved using cellulose tris (3,5-dimethylphenylcarbamate) (Lux Cellulose-1) and $n$-Hex/ IPA $90: 10$ or amylose tris (3,5-dimethylphenylcarbamate) (Lux Amylose-2) and MeCN/IPA 90:10. The other combinations of mobile/stationary phases tested were found to be unsuitable for resolving the samples tested.
Considering both systems (Lux Cellulose- 1 with $n$-Hex/ IPA 90:10 and Lux Amylose-2 with MeCN/IPA 90:10) showed a comparable chiral resolving ability, the criteria used to discriminate between methods was the solubility of the analytes in the respective mobile phases. Indeed, since the solubility of the racemates $( \pm)-4 \mathrm{a}-\mathrm{c}$ was poorer in $n$-Hex/IPA 90:10 than in MeCN/IPA 90:10, the latter method was chosen in order to increase the amount of analyte injected for each separation cycle and to reduce the times of separations. Once optimized, these analytical conditions were applied to the semipreparative system. Separations were performed on Lux Amylose-2 column (250 $\mathrm{mm} \times 4.6 \mathrm{~mm}$ I.D., $5 \mu \mathrm{m}$ ) (Fig. 3) and were suitable to collect the required amounts of pure enantiomers (-)-4a-c and (+)-4a-c for biological studies. Finally, the fractions collected were analyzed in the analytical system using Lux Amylose-2 column and the aforementioned conditions to determine the enantiomeric excess (ee) of pure isomers, which resulted in $e e$ values between $98.1 \%$ and $99.9 \%$ (Fig. 4).

\section{Configurational Assignment}

In order to establish absolute configuration of optically active compounds in the series, X-ray analysis was performed. Considering the difficulties in obtaining suitable crystals of all enantiomers, one isomer for each pair was processed for X-ray analysis, as reported for (-)-4a, (+)-4b and (-)-4c (Fig. 5) in the experimental section. Assignment of the absolute configurations at C5 was then unambiguously established for all of the enantiomers by a combination of crystallographic data, polarimetric analysis, and comparative elution order determined by chiral HPLC separations.

Among the three analyzed enantiomers, compound (-)-4a contains a bromine as a heavy atom that was useful in determining the exact configuration using the Flack parameter derived from X-ray analysis. ${ }^{24}$ For enantiomers (+)-4b and $(-)-4 \mathrm{c}$, the absolute configurations at $\mathrm{C} 5$ of the pyridazine ring could not be determined positively by using only Flack parameters, as these compounds don't contain heavy atoms in their structures. Thus, these crystallographic results needed to be supported by a correlation with both polarimetric and chiral chromatographic analysis. Chiral HPLC and polarimetric analysis indicated that racemates $( \pm)$-4a-c have same-sense chiral recognition mechanism and, in all cases, the first eluted enantiomer on Lux Amylose-2 column rotated the polarized light in the negative direction (Figs. 3 and 4). Since the chiral stationary phases are given just in one configuration, enantiomer elution order is a very important aspect in the study of enantiorecognition mechanisms. ${ }^{25}$ In a series of analogue compounds, such as in our case, the similarity of both skeleton and
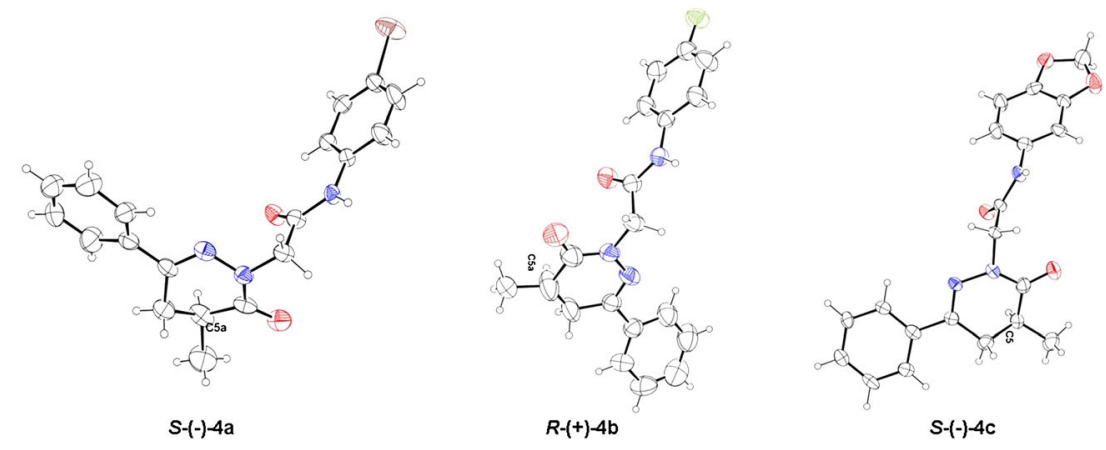

Fig. 5. An ORTEP view of the molecular structure of $S-(-)-4 \mathrm{a}, R-(+)-4 \mathrm{~b}$, and $S-(-)-4 \mathrm{c}$. 
TABLE 2. Analysis of FPR activation by racemates ( \pm )-4a-c, pure enantiomers $S-(-)-4 a-c / R-(+)-4 a-c$, and non-chiral analogues 8a-c in HL-60 cells expressing human FPR1, FPR2, or FPR3

\begin{tabular}{lccc}
\hline & \multicolumn{3}{c}{$\mathrm{Ca}^{2+}$ Mobilization $\mathrm{EC}_{50}(\mu \mathrm{M})$ and Efficacy (\%) } \\
\cline { 2 - 4 } compd & FPR1 & FPR2 & FPR3 \\
\hline$( \pm)-4 \mathrm{a}$ & $23.5(55)$ & $7.0(65)$ & N.A. \\
$S-(-)-4 \mathrm{a}$ & $19.5(75)$ & $10.7(95)$ & $8.3(40)$ \\
$R-(+)-4 \mathrm{a}$ & $24.4(80)$ & $10.0(85)$ & $7.1(40)$ \\
$8 \mathrm{a}$ & $21.5(50)$ & $10.1(45)$ & NA \\
$( \pm)-4 \mathrm{~b}$ & N.A. & N.A. & N.A. \\
$S-(-)-4 \mathrm{~b}$ & N.A. & N.A. & N.A. \\
$R-(+)-4 \mathrm{~b}$ & N.A. & N.A. & N.A. \\
$8 \mathrm{~b}$ & N.A. & N.A. & N.A. \\
$( \pm)-4 \mathrm{c}$ & N.A. & N.A. & N.A. \\
$S-(-)-4 \mathrm{c}$ & N.A. & N.A. & N.A. \\
$R-(+)-4 \mathrm{c}$ & N.A. & N.A. & N.A. \\
$8 \mathrm{c}$ & N.A. & N.A. & N.A. \\
\hline
\end{tabular}

${ }^{a}$ N.A., no activity was observed (no response was observed during first 2 min after addition of compounds under investigation) considering the limits of efficacy $>20 \%$ and $\mathrm{EC}_{50}<50 \mu \mathrm{M}$. The $\mathrm{EC}_{50}$ values are presented as the mean of three independent experiments, in which median effective concentration values $\left(\mathrm{EC}_{50}\right)$ were determined by nonlinear regression analysis of the dose-response curves (5-6 points) generated using GraphPad Prism 5 with 95\% confidential interval ( $p$ 0.05). Efficacy (in bracket) is expressed as percent of the response induced by 5 nM fMLF (FPR1) or 5 nM WKYMVm (FPR2 and FPR3).

substituents on a chiral center are the main determinant in retaining the same elution order for enantiomers having the same absolute configuration and, obviously, the same optical rotatory sign. X-ray data of (-)-4a univocally indicated an (S)-absolute configuration for this isomer. Thus, we could confirm that all enantiomers (-)-4a-c were in the (S)-form and that the $(R)$-form could be assigned to the corresponding dextrorotatory isomers (+)-4a-c. Absolute configurations were then unambiguously assigned as follows for the three enantiomeric pairs of the series: $S-(-)-4 \mathrm{a}-\mathrm{c}$ and $R-(+)-4 \mathrm{a}-\mathrm{c}$. Furthermore, the polarimetric and crystallographic data presented in this study may be useful to assign the absolute configuration of compounds having a similar structure to that of $( \pm)-4 a-c$.

\section{Pharmacology}

The final racemates $( \pm)-4 \mathrm{a}-\mathrm{c}$, the pure enantiomers $S$ - $(-)$ $4 \mathrm{a}-\mathrm{c} / R-(+)-4 \mathrm{a}-\mathrm{c}$, and the non-chiral analogues $8 \mathrm{a}-\mathrm{c}$ were tested for their ability to induce intracellular $\mathrm{Ca}^{2+}$ flux in HL-60 cells transfected with FPR1, FPR2, or FPR3, in order to evaluate their ability to act as FPR agonists. ${ }^{26,27}$ As shown in Table 2, biological assays revealed that the bromo derivatives $( \pm)-4 \mathrm{a}$, $S-(-)-4 \mathrm{a}, R-(+)-4 \mathrm{a}$ and $8 \mathrm{a}$ activated FPR1 and FPR2 with $\mathrm{EC}_{50}$ values in a similar micromolar range, whereas they were slightly more potent FPR2 agonists, with $\mathrm{EC}_{50}$ values between 7.0 and $10.7 \mu \mathrm{m}$. Moreover, enantiomers $S$-(-)-4a and $R-(+)-4$ a also exhibited an appreciable agonistic activity for $\mathrm{FPR} 3$, with $\mathrm{EC}_{50}$ values in the low micromolar range $\left(\mathrm{EC}_{50}=\right.$ 8.3 and $7.1 \mu \mathrm{m}$ respectively). As reported in Table 2 , all other compounds bearing a fluorine or a methylenedioxy side chain were inactive. To confirm specificity of the observed FPR agonist activity, the active compounds were also evaluated in WT cells (wild-type non transfected HL-60 cells) and were found to be inactive.

\section{CONCLUSIONS}

Taken together, our data confirmed that the stereogenic center at $\mathrm{C} 5$ of the pyridazine ring plays only a marginal role in the activity and selectivity of the FPR agonists. On the other hand, the $N$-bromophenylacetamide moiety on the lactam nitrogen of the heterocyclic scaffold seems to play a major role in agonist activity, which is in agreement with previously reported agonists. ${ }^{7,11}$ Considering that pyridazine-based compounds represent good candidates for FPR-specific agonists, further studies are in progress to evaluate the influence of stereochemistry in other experimental in vitro models such as chemotaxis and neutrophils activation.

\section{ACKNOWLEDGMENTS}

This work was supported in part by National Institutes of Health grant GM103500, an equipment grant from the M.J. Murdock Charitable Trust, and the Montana State University Agricultural Experimental Station.

\section{LITERATURE CITED}

1. Beutler B. Innate immunity: An overview. Mol Immunol 2004;40:845-859.

2. Serhan CN, Brain SD, Buckley CD, Gilroy DW, Haslett C, O'Neill LA, Perretti M, Rossi AG, Wallace JL. Resolution of inflammation: state of the art, definitions and terms. FASEB J 2007;21: 325-332.

3. Gilroy DW, Lawrence T, Perretti P, Rossi AG. Inflammatory Resolution: new opportunities for drug discovery. Nat Rev Drug Discov 2004;3:401-416.

4. Zhang L, Falla TJ. Host Defense Peptides for Use as Potential Therapeutics. Curr Opin Invest Drugs 2009;10:164-171.

5. Dufton N, Perretti M. Therapeutic anti-inflammatory potential of formylpeptide receptor agonists. Pharmacol Ther 2010;127:175-188.

6. Ye RD, Boulay F, Wang JM, Dahlgren C, Gerard C, Parmentier M, Serhan $\mathrm{CN}$, Murphy PM. International Union of Basic and Clinical Pharmacology. LXXIII. Nomenclature for the formyl peptide receptor (FPR) family. Pharmacol Rev 2009;61:119-161.

7. Cilibrizzi A, Quinn MT, Kirpotina LN, Schepetkin IA, Holderness J, Ye $\mathrm{RD}$, Rabiet MJ, Biancalani C, Cesari N, Graziano A, Vergelli C, Pieretti S, Dal Piaz V, Giovannoni MP. 6-methyl-2,4-disubstituted pyridazin-3 (2H)-ones: a novel class of small-molecule agonists for formyl peptide receptors. J Med Chem 2009;52:5044-5057.

8. Owings FF, Fox M, Kowalski CJ, Baine NH. An enantioselective synthesis of SK\&F93505, a key intermediate for preparing cardiotonic agents. J Org Chem 1991;56:1963-1966.

9. Nomoto Y, Takai H, Ohno T, Nagashima K, Yao K, Yamada K, Kubo K, Ichimura M, Mihara A, Kase H. Studies of Cardiotonic Agents. 8. Synthesis and Biological Activities of Optically Active 6-(4 (Benzylamino)-7quinazolinyl)-4,5-dihydro-5-methyl-3(2H)-pyridazinone (KF15232). J Med Chem 1996;39:297-303.

10. Lues I, Beier N, Jonas R, Klockow M, Haeusler G. The two mechanisms of action of racemic cardiotonic EMD 53998, calcium sensitization and phosphodiesterase inhibition, reside in different enantiomers. J Cardiovasc Pharmacol 1993;21:883-892.

11. Cilibrizzi A, Schepetkin IA, Bartolucci G, Crocetti L, Dal Piaz V, Giovannoni MP, Graziano A, Kirpotina LN, Quinn MT, Vergelli C. Synthesis, Enantioresolution, and Activity Profile of Chiral 6-Methyl-2,4Disubstituted Pyridazin-3 $(2 H)$-ones as Potent $N$-Formyl Peptide Receptor Agonists. Bioorg Med Chem 2012;20:3781-3792.

12. Serhan CN, Maddox JF, Petasis NA, Akritopoulou-Zanze I, Papayianni A, Brady HR, Colgan SP, Madara JL. Design of lipoxin A4 stable analogs that block transmigration and adhesion of human neutrophils. Biochemistry 1995;34:14609-14615.

13. Takano T, Fiore S, Maddox JF, Brady HR, Petasis NA, Serhan CN. Aspirin-triggered 15-epi-lipoxin A4 (LXA4) and LXA4 stable analogues are potent inhibitors of acute inflammation: evidence for anti-inflammatory receptors. J Exp Med 1997;185:1693-1704.

14. Frohn M, Xu H, Zou X, Chang C, McElvain M, Plant MH, Wong M, Tagari $P$, Hungate R, Bürli RW. New 'chemical probes' to examine the role of the hFPRL1 (or ALXR) receptor in inflammation. Bioorg Med Chem Lett 2007;17:6633-6637. 
15. Hu W, Ranaivo RH, Roy SM, Behanna HA, Wing LK, Munoz L, Guo L, Van Eldik LJ, Watterson DM. Development of a novel therapeutic suppressor of brain proinflammatory cytokine up-regulation that attenuates synaptic dysfunction and behavioral deficits. Bioorg Med Chem Lett 2007;17:414-418.

16. Wermuth CG, Leclerc G. Pyridazine derivatives of therapeutic interest. VI. Synthesis of morpholinoethyl-4-methyl-6-phenyl-3-pyridazone (Ag 246) analogs modified in the aminoalkyl side chain. Chim Ther 1970;5:243-246.

17. Walker N, Stuart D. Correction for Lorentz and polarization effects. Acta Cystallogr Sect A 1983;39:158166.

18. Sheldrick GM. SADABS version 2.03: a Program for Empirical Absorption Correction; Universität Göttingen, 1997-2001.

19. Burla MC, Calandro R, Cavalli M, Carrozzini B, Cascarano GL, De Caro L, Giacovazzo C, Polidori G, Spagna R. SIR2004: an improved tool for crystal structure determination and refinement. J Appl Cryst 2005;38:381-388.

20. Sheldrick GM. SHELXL97: Program for crystal structure refinement. Göttingen, Germany: Institut für Anorganische Chemie de Universitat Göttingen; 1997.
21. Christophe T, Karlsson A, Rabiet MJ, Boulay F, Dahlgren C. Phagocyte Activation by Trp-Lys-Tyr-Met-Val-Met, Acting Through FPRL1/LXA4R, Is Not Affected by Lipoxin A4. Scand J Immunol 2002;56:470-476.

22. Schepetkin IA, Kirpotina LN, Khlebnikov AI, Quinn MT. Highthroughput screening for small-molecule activators of neutrophils: identification of novel $N$-formyl peptide receptor agonists. Mol Pharmacol 2007;71:1061-1074.

23. Breukelman SP, Meakins GD, Roe AM. Preparation and some reactions of 4- and 5-aryl-4,5-dihydropyridazin-3(2H)-ones. J Chem Soc, Perkin Trans1 1985;8:1627-1635.

24. Flack HD, Bernardinelli G. The use of X-ray crystallography to determine absolute configuration. Chirality 2008;20:681-690.

25. Okamoto M. Reversal of elution order during the chiral separation in high performance liquid chromatography. J Pharm Biom Anal 2002;27:401-407.

26. Didsbury JR, Uhing RJ, Tomhave E, Gerard C, Gerard N, Snyderman R. Functional high efficiency expression of cloned leukocyte chemoattractant receptor cDNAs. FEBS Lett 1992;297:275-279.

27. Prossnitz ER, Quehenberger O, Cochrane CG, Ye RD. Transmembrane signalling by the $N$-formyl peptide receptor in stably transfected fibroblasts. Biochem Biophys Res Commun 1991;179:471-476. 